\title{
Hölderlin-Ausstellung und Begleitprogramm
}

\section{Oktober 2020 bis April 2021}

\section{Eröffnung der Ausstellung}

Am 12. Oktober 2020 ist die Jubiläumsausstellung anlässlich des 250. Geburtstages Friedrich Hölderlins eröffnet worden, unmittelbar nach der Inbetriebnahme des Neubaus. Bis kurz vor der Eröffnung liefen die vielschichtigen Vorbereitungen, die von der Übergabe und Bauabnahme abhängig waren und deshalb nicht den geplanten längeren Vorlauf haben konnten. Nur dank des großen Einsatzes aller Beteiligten war am Eröffnungstag der Aufbau pünktlich abgeschlossen. Die feierliche Eröffnung am Abend durch die Ministerin für Wissenschaft, Forschung und Kunst, Frau Theresia Bauer (Abb. 1), war überaus gelungen

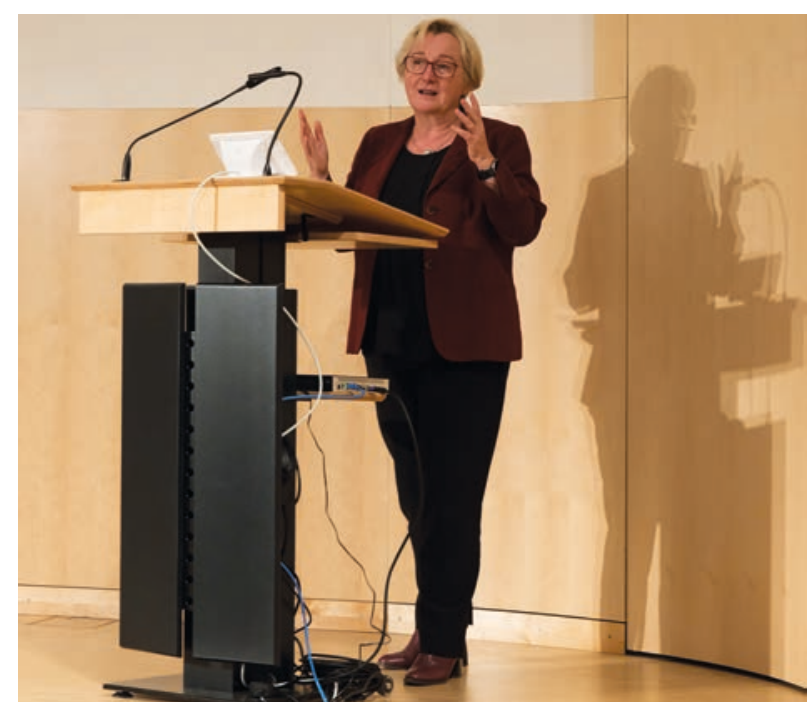

Abb. 1

und wurde nur von den Corona bedingten Beschränkungen der Gästeliste (nur maximal 37 Gäste waren zugelassen) getrübt. Die Festrede hielt Prof. Thomas Knubben zum Thema "Hölderlin 21". Das musikalische Programm in Kooperation mit der Internationalen Hugo-Wolf-Akademie wurde von Alexander Fleischer (Klavier) und Nikola Diskić (Bariton) gestaltet. Im Anschluss an den offiziellen Eröffnungsteil führte der Kurator Dr. Jörg Ennen durch die Ausstellung (Abb. 2 und 3) und der Direktor der WLB, Dr. Rupert Schaab, durch den Erweiterungsbau (Abb. 4), worauf noch ein kleiner Stehempfang folgte.

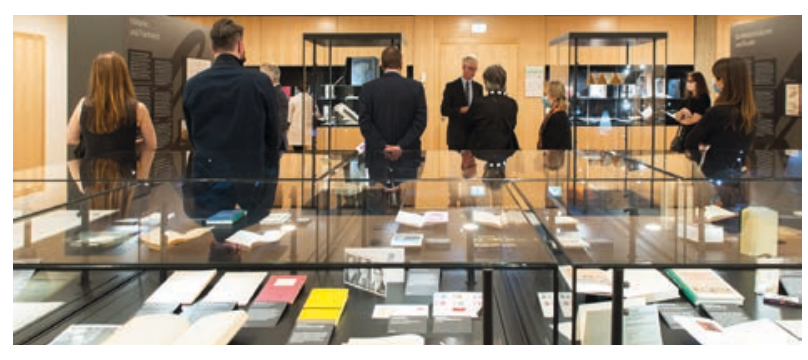

Abb. 2

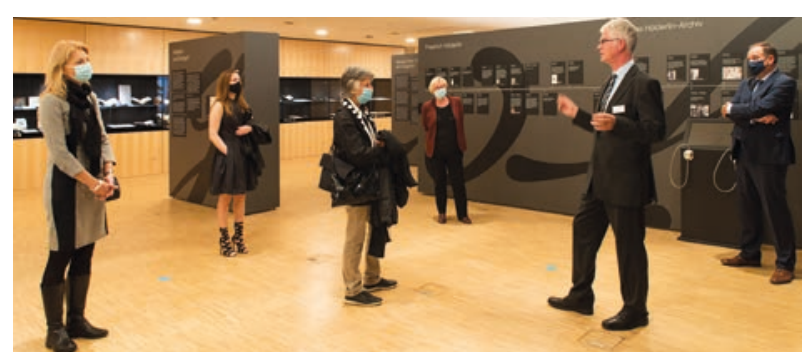

Abb. 3

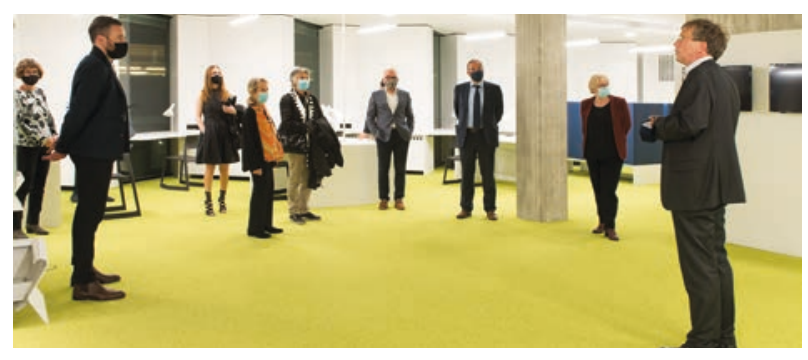

Abb. 4

\section{Podiumsdiskussion}

Eine Woche nach der Eröffnung waren Karl-Heinz Ott und Wolfgang Koch zu Gast in der WLB, um in einer Podiumsdiskussion über das aktuelle Buch von Ott "Hölderlins Geister" zu diskutieren (Abb. 5). ${ }^{1}$ Karl-Heinz Otts Buch war 2020 gemäß den Ausleihzahlen der WLB das am stärksten nachgefragte Hölderlin-Buch neben der Biografie von Rüdiger Safranski. Das große Interesse spiegelte sich auch im Vorfeld der Veranstaltung wider: Ohne die Teilnehmerbeschränkungen wären sicherlich ein Vielfaches der Besucher*innen gekommen. Die Corona bedingte Beschränkung der Besucherzahl wurde durch das neu eingerichtete Buchungssystem gesteuert. von Maik Bozza: https://www.wlb-stuttgart.blog/hoelderlins-geister/. 


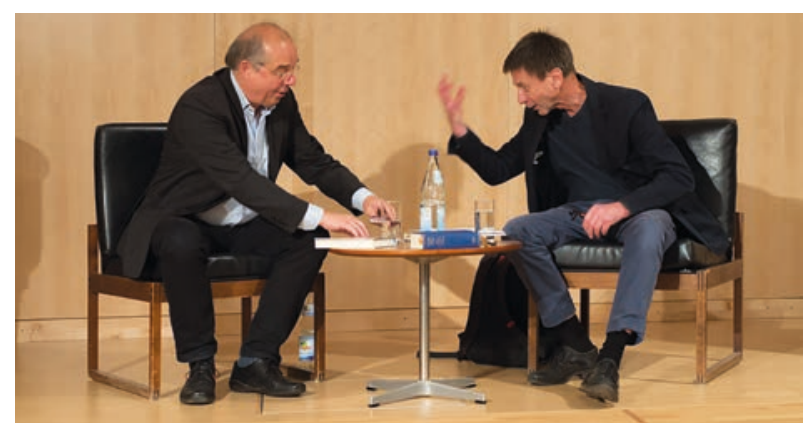

Abb. 5

\section{Performance}

Am 27. Oktober, gerade noch vor dem erneuten „kulturellen Lockdown", fand eine bemerkenswerte Performance statt, in der Malerei, Musik und Rezitation in einen lebendigen Dialog traten. Die Veranstaltung war Teil des „Literatursommers 2020". In diesem Jahr stand das beliebte Förderprogramm der Baden-Württemberg Stiftung unter dem Motto: „Hölderlin und Hegel. 250 Jahre Sprache

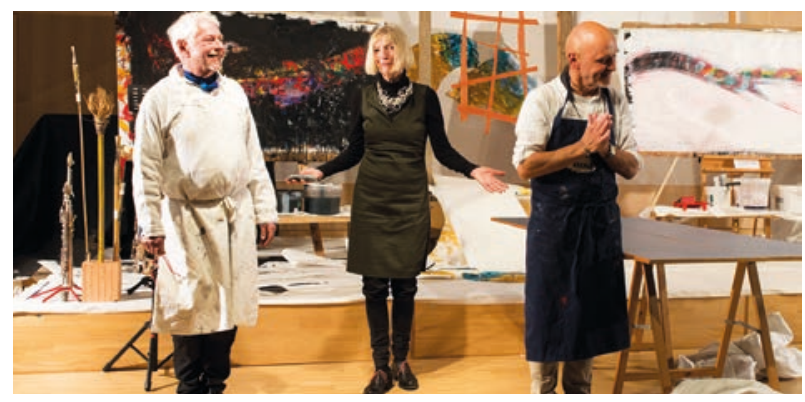

Abb. 6

und Vision“. Dem Künstlerteam (Abb. 6) um Werner Englert (Musik), Katharina Hoehler (Malerei) und Dieter E. Neuhaus (Dramaturgie) ist es eindrucksvoll gelungen, dieses Motto in einer innovativen und experimentellen Performance umzusetzen. ${ }^{2}$

\section{Lockdown}

Leider musste die Ausstellung einschließlich Begleitprogramm nach dem erfolgreichen Start infolge der rasant steigenden Infektionszahlen bereits ab November wieder auf unbestimmte Zeit schließen.

\section{Verlängerung der Ausstellung}

Schnell zeichnete sich ab, dass der Lockdown bis zum Ende der regulären Ausstellungslaufzeit (10. Januar) anhalten würde. Glücklicherweise ist es gelungen, durch mehrere organisatorische Maßnahmen die Hölderlin-Ausstellung noch bis zum 30. April 2021 zu verlängern. Das ausgefallene Begleit- programm versuchte man so weit wie möglich in den Frühling 2021 zu verschieben, um eine letzte Chance der Realisierung zu nutzen. Auch offiziell wurde das Hölderlinjahr bis Juni 2021 verlängert und das Logo entsprechend angepasst.

\section{Wiedereröffnung}

Ab Mitte Februar begannen die Vorbereitungen für eine Wiedereröffnung der Ausstellung, um optimal vorbereitet zu sein und schnell reagieren zu können, sobald die Inzidenzwerte eine Öffnung im März nahe an Hölderlins Geburtstag - möglich machen sollten. Da die weitere Entwicklung des Infektionsgeschehens kaum vorhersehbar war, mussten alle Szenarien mit einkalkuliert werden. Instrumente der Öffentlichkeitsarbeit wurden mobilisiert, Plakate und Flyer entsprechend aktualisiert, um sie kurzfristig in den Umlauf bringen zu können. Am 8. März war es dann soweit: Die Ausstellung konnte nach gut vier Monaten ihre Türen wieder öffnen.

\section{Begleitprogramm}

Mit der Vorbereitung der Wiedereröffnung der Ausstellung setzte man auch einen Großteil der Begleitveranstaltungen neu an, wohlwissend, dass es völlig ungewiss sein würde, inwieweit die Infektionszahlen eine Realisierung zuließen. Wöchentlich - vom 31. März bis 28. April - wurde den Besucherinnen und Besuchern neben der Ausstellung eine interessante Veranstaltung angeboten:

\section{März, 18 Uhr:}

Friedrich Hölderlin / DICHTER SEIN. UNBEDINGT Filmabend mit dem Regisseur Rolf Lambert 9. April, 18 Uhr:

Rezitationsabend der WLB in Kooperation mit der Akademie für gesprochenes Wort

14. April, 18 Uhr:

Hans Gerhard Steimer: Friedrich Hölderlins Homburger Folioheft in diachroner Darstellung

20. April, 17 Uhr und 19 Uhr:

Vertonungen von Friedrich Hölderlin.

Ein Liederabend in Kooperation mit der Internationalen Hugo-Wolf-Akademie 28. April, 18 Uhr:

Der Not ist jede Lust entsprossen - ein Konzertabend auf Spuren Hölderlins - mit Anne Schneider (Gesang), Urs Stämpfli (Schauspiel),

Astrid Alexander (Audio). 